\title{
Clinical operational considerations and responses to lockdown and reopening in the Covid-19 pandemic: experience of a tertiary ophthalmology centre in Singapore
}

\author{
Hou Boon Lim ${ }^{1,2} \cdot$ Tien-En Tan ${ }^{1,2} \cdot$ Beau J. Fenner ${ }^{1,2} \cdot$ Jenny Heah ${ }^{1} \cdot$ Anshu Arundhati ${ }^{1,2,3} \cdot$ Wei Boon Khor ${ }^{1,2,4}$. \\ Pui Yi Boey ${ }^{1,2,5} \cdot$ Shu Yen Lee ${ }^{1,2} \cdot$ Edmund Y. M. Wong ${ }^{1,2} \cdot$ Tien Yin Wong $\mathbb{D}^{1,2}$
}

Received: 3 December 2020 / Revised: 4 March 2021 / Accepted: 15 April 2021 / Published online: 28 September 2021

(c) The Author(s), under exclusive licence to The Royal College of Ophthalmologists 2021

\begin{abstract}
Background Much has been written on infection control and clinical measures for ophthalmic institutions and departments to cope with the COVID-19 pandemic. However, few articles have detailed implementation plans to manage lockdowns and subsequent re-openings. In this article, specific operational responses and their outcomes in a large tertiary ophthalmology centre are described.

Method Through a concerted effort led by a dedicated task force, the Singapore National Eye Centre (SNEC) planned and executed an operational transformation to respond to the restrictions imposed on healthcare delivery during a national lock down. A carefully calibrated re-starting of services was carried out with the subsequent phased reopening of the country, taking into consideration unique constraints faced at that time. Strategies for operating in the new normal environment were also developed.

Results Outpatient attendances were safely and expediently reduced by $70 \%$ (8749 vs. 29,311$)$ and $82 \%$ (5164 vs. 29,342$)$ in April and May 2020, respectively, compared to the corresponding months in 2019. A correspondingly large reduction in surgical load was also achieved through a similar triaging and prioritization system. Through optimizing the center's use of space and time, as well as expanding on new models of care, a return to pre-pandemic patient load was achieved 3 months into the phased reopening of the country, and subsequently exceeded in the following months.

Conclusion The lessons gleaned from SNEC's experience may be useful for institutions currently facing the same challenges, and for future responses to COVID-19 resurgences or other pandemics.
\end{abstract}

\section{Introduction}

The first case of Coronavirus disease 2019 (COVID-19) was reported in December 2019 in Wuhan, China. In the months that followed, COVID-19 was declared a global

Tien Yin Wong

wong.tien.yin@singhealth.com.sg

1 Singapore Eye Research Institute, Singapore National Eye Centre, Singapore, Singapore

2 Duke-National University of Singapore Medical School, Singapore, Singapore

3 SNEC Eye Clinic, Changi General Hospital, Singapore, Singapore

4 SNEC Eye Clinic, Sengkang General Hospital, Singapore, Singapore

5 SNEC Eye Clinic at Bedok, Singapore, Singapore pandemic by the World Health Organization, and as of 29 January 2021, the virus has infected at least 101 million people and is responsible for more than 2.1 million deaths worldwide [1, 2].

Singapore had its first case of COVID-19 on 23 January 2020 [3]. Sharp increases in the number of cases in Singapore led to a national lockdown from 7 April 2020 to 1 June 2020 [4], followed by a phased resumption of social and economic activities thereafter. As the largest tertiary ophthalmology referral centre in the country, the Singapore National Eye Centre (SNEC) underwent a large-scale operational transformation to deal with the unique circumstances and constraints. With more than 80 ophthalmologists on faculty and 1000 staff members, SNEC provides more than $50 \%$ of public sector eye care across a network of 9 other satellite clinics and manages a caseload of approximately 340,000 patient visits annually. 
In this article, we describe in detail the key considerations and the clinical implementation of a broad range of operational changes in our institution through the phases of COVID-19: lockdown, reopening and planning for the new normal.

\section{General measures}

General measures and heightened infection control practices were put in place in SNEC from January 2020, in line with other ophthalmic institutes in Singapore, and globally. These measures have been previously described in detail [5-15].

\section{Lockdown: clinical operational considerations and response}

In an effort to curb community transmission of COVID-19, the Singapore government imposed a nationwide lockdown on 7 April 2020. A wide range of restrictions on population movement and social gatherings were enforced, and only the obtaining or provision of essential services was permitted to continue. In healthcare, the MOH defined routine ophthalmology services as "non-essential", and all elective eye procedures, including surgeries for stable cataracts, were deferred [16]. Provision of services for conditions that required immediate care to prevent imminent loss of vision, however, was allowed to continue.

The implications of these regulations on visits to SNEC were two-fold: First, patients were not allowed to leave their homes to attend routine non-urgent follow-up appointments, as these were considered non-essential. Second, doctors were not allowed to go to work other than for managing patients with urgent conditions. To mitigate these changes, the SNEC Disease Outbreak Task Force (DOTF) set about planning for the re-organizing of the centre, and the rescheduling large numbers of patient appointments, with the following overall priorities: (1) meeting the government's intent of minimizing population movement; (2) maintaining the standard of ophthalmic care; and (3) maximizing overall patient and staff safety. This dedicated task force, constituted by senior management members and key stakeholders in medical, nursing, ancillary staff, human resource and operations, oversaw the implementation of the operational transformation of SNEC's outpatient clinics, operating theatres and surgical facilities, as well as staffing and overall model of ophthalmic care provision.

\section{Outpatient clinic visits and appointments}

Working with our clinical services and resource management teams, doctors reviewed the electronic medical records of every scheduled patient to triage and determine the urgency of their upcoming appointment using guidelines provided by the DOTF. These were based on (1) diagnosis; (2) treatment and stability of condition; and (3) time of the last review. While all doctors were involved in the triaging of patients from our general outpatient clinics, patients with more complex issues were only triaged by ophthalmologists from the respective subspecialties. Other than for patients with imminently vision-threatening conditions, all other appointments were deferred. An appropriate timeline for rescheduling, ranging between 3 and 24 months, was indicted by the doctor using a standardized template. These were then collated and effected by the resource management team. Mobile phone text messages were sent to inform patients of the changes to their appointments, and bridging prescriptions for medications (e.g., topical glaucoma medications) provided when required. A free-of-charge medication delivery service was instituted to allow patients to stay at home. Fast-track approval was given for staff access to the hospital's intranet via a secured virtual private network to allow most of this work to be done from home.

Although the nationwide partial lockdown started on 7 April, the closure of routine clinics was put into effect from 13 April as time was needed for guidance from the ministry, and for planning and preparation work to be completed. From 7 to 13 April, a substantial decline in patient attendance for non-urgent appointments was observed as patients complied with the lockdown restrictions. Overall, SNEC was able to expediently reduce the number of attendances by $70 \%$ ( 8749 vs. 29,311$)$ and $82 \%(5164$ vs. 29,342$)$ in April and May 2020, respectively, compared to the corresponding months in 2019 (Fig. 1). Amongst the different sub-specialty clinics, our paediatric ophthalmology clinic achieved the highest reduction of 94\% in May 2020, while the retina clinic achieved the least reduction at $74 \%$ in April 2020 (Fig. 2).

To track the patients with deferred appointments, we tagged each case with a unique identifier in the appointment system. This allowed us to avoid further rescheduling of the same visit and enabled the re-creation of early appointments once lockdown measures were eased. Anticipating the possibility of extended or reintroduced lockdown after reopening, for certain subspecialties, triaging of patients after each consult was also initiated to indicate a suitable deferral period if deferment was again required in the future.

\section{Operating theatres and surgery}

A similar triaging process was adopted for the management of scheduled surgeries based on a priority system (Table 1). Patients who had developed, or were at immediate risk of developing, sight-threatening complications from their ocular conditions were considered urgent. For such 
Fig. 1 Comparison between patient attendances in 2019 and 2020. Light and dark bars: attendances in 2019 and 2020, respectively. Percentages:

Number of attendances in 2020

as a proportion of number in 2019.
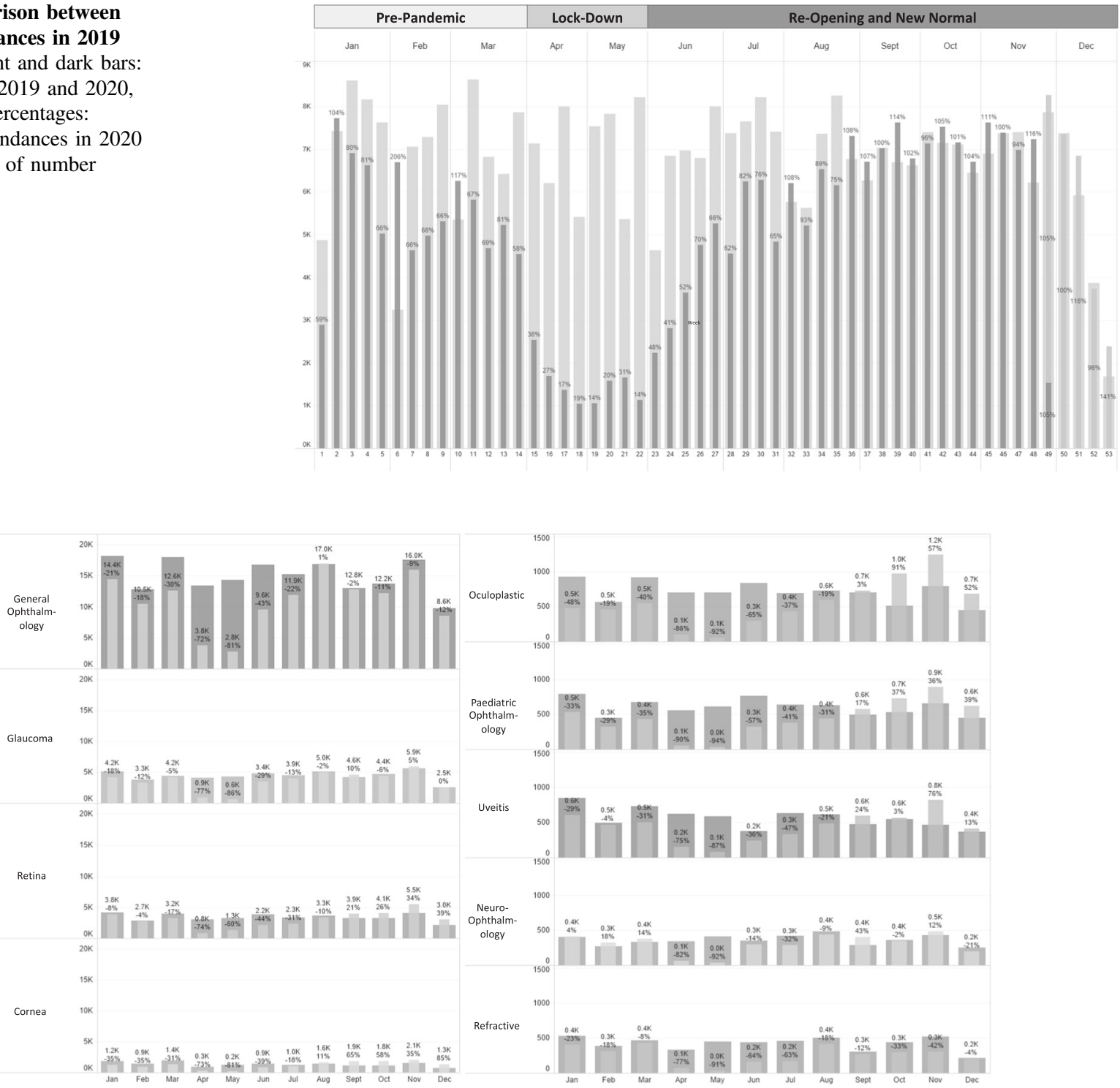

Fig. 2 Comparison between patient attendances of subspeciality services in 2019 and 2020. Light and dark bars: attendances in 2020 and 2019 respectively. Percentages: Number of attendance in 2020 as a proportion of number in 2019).

conditions, surgeries proceeded as planned within 1-2 days. For less urgent, but still potentially vision-threatening cases, surgery was arranged to be performed within 1-2 months. The majority of previously listed elective surgeries were for non-urgent conditions and were therefore postponed. With significantly reduced surgical numbers (Fig. 3), only two operating theatres remained operational (down from the usual 10 active theatres) to minimize nursing and anaesthetic staffing requirements.

\section{Reconfiguration of outpatient clinics}

With the drastically reduced patient load, multiple simultaneously running clinics within each subspecialty service were collapsed into single team-based clinics to care for patients whose appointments could not be postponed. It followed that patients were not typically reviewed by their usual care provider because of this reorganization. From 11 clinical areas of various sizes, these were merged into 7 smaller areas from mid-April and further reduced to 5 in May. By merging clinical areas, the deployment of nursing, allied health and ancillary staff was optimized, allowing more of them to remain at home. Safe distancing was maintained given the significantly reduced patient attendances.

Maintaining patient access to appropriate emergency ophthalmic care was an important priority for SNEC, and a medical team supervised by senior faculty was deployed to 
cover the center's Acute Care Clinic (ACC). The ACC is a "walk-in" service to cater to patients with urgent ocular complaints and is normally run by residents. It was observed that attendance at ACC increased by 50\% during the lockdown period after an initial reduction in early April. Many attendances were by patients who were unaware of changes to their appointment date, as well as those who presented despite receiving notifications due to concerns over their ocular conditions.

\section{Re-opening: clinical operational considerations and response}

By 19 May, widespread community transmission of COVID-19 had declined, and the government announced that the lockdown would end on 1 June, with the phased resumption of activities commencing on 2 June. After significantly scaling back outpatient clinical and surgical services during the lockdown, and with deferral of a large volume of outpatient visits and elective surgical cases, managing the resultant backlog of clinical workload and reopening the center's clinical services safely, in compliance with the government's restrictions, posed its own set of unique operational challenges.

Restrictions on the delivery of healthcare services were lifted in a graduated manner that corresponded to the phased reopening of the country. An immediate return to businessas-usual in SNEC was not possible due to two key factors. First, the need for continued safe-distancing measures in the centre meant that clinics were not able to physically accommodate the same number of patients as they did previously. Second, a significant number of medical and ancillary staff was still committed to national COVID-19 operations external to SNEC, and manpower became a significant limiting factor to return fully to routine operations. To mitigate these limitations, a plan was developed by the task force to increase clinic and surgical workload in a step-wise manner, achieving an initial target of $73 \%$ of the workload by July 2020, compared to the corresponding month in 2019 (23,531 vs. 32,311 attendances), returning to pre-pandemic workload levels by August 2020, and exceeding it in the following months (Figs. 1 and 3 ).

\section{Maximizing space use}

To accommodate the highest possible number of patients in the clinic and day-surgery ward at any one time while meeting safe distancing requirements, usage of the preexisting physical space was maximized through layout reconfiguration and adding more seats for patients wherever possible. New spaces were also identified and converted into temporary patient holding areas (Figs. 4-6). The 
Fig. 3 Number of surgeries performed in SNEC in 2020.

Dark bars: Surgeries performed. Light bars: surgeries scheduled.

Fig. 4 Utilization of SNEC's central atrium as the patient waiting area. Chairs on the left serve as holding areas for patients waiting to collect medications at the pharmacy (not visible in photo) while chairs on right cater to patients registering for consultation.
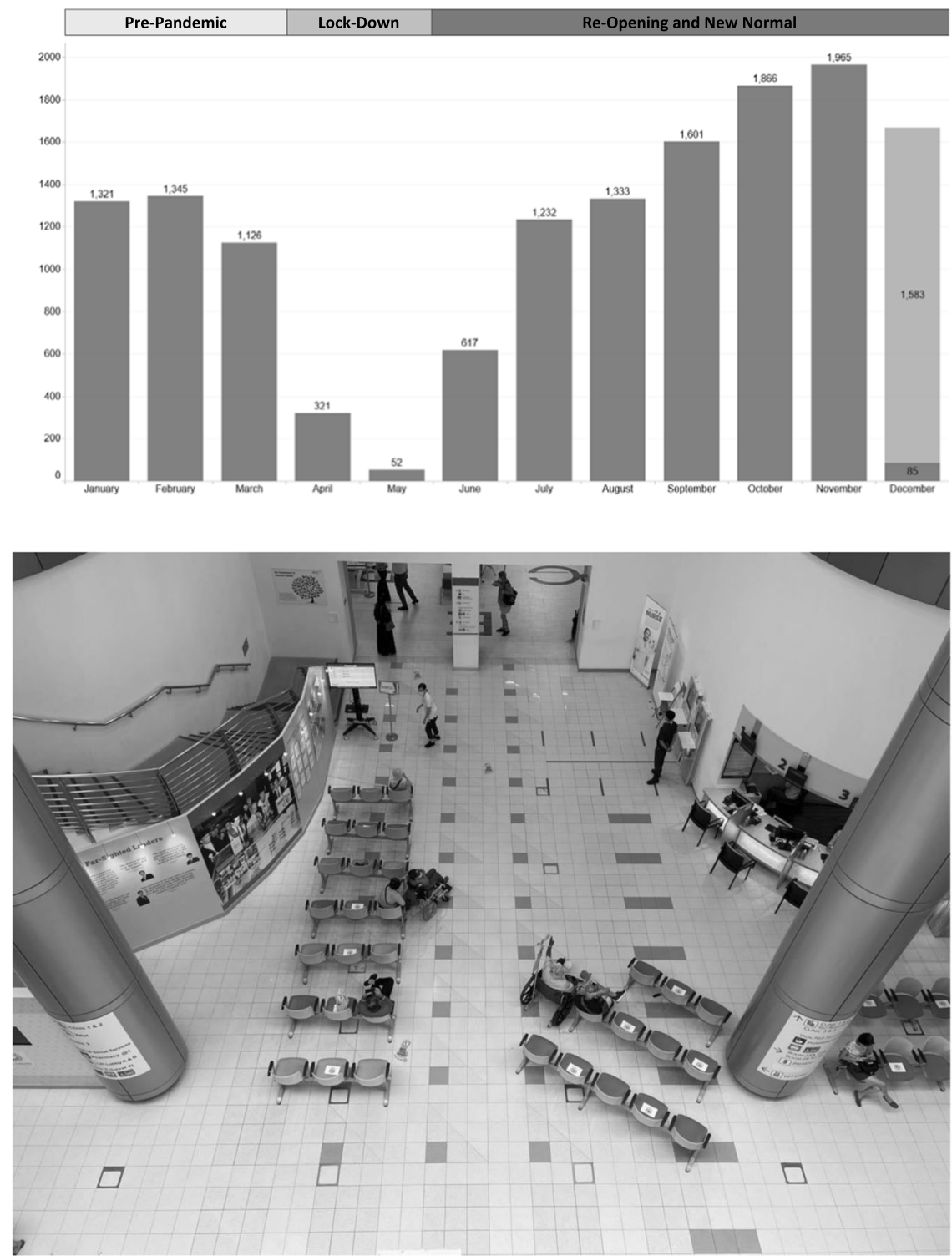

maximum number of patient appointments allowable per session in each clinic was then calculated based on the number of waiting area seats, an estimated number of accompanying caregivers (for patients without mobility problems or need for special assistance, only one accompanying person was permitted), as well as the average turnover rate of patients in the clinics. Appointments beyond the designated thresholds in each clinic session were rescheduled or deferred based on triaged clinical need.

Measures to reduce patients' dwell time in the centre were also implemented. This included adequately staffing clinics with doctors to reduce consultation wait times, minimizing consultation times, avoiding unnecessary ad hoc ophthalmological tests, home delivery of medications, and remote payment and appointment scheduling. These measures enabled patients to shorten the total time they needed to spend in the centre and increased the turnover rate in waiting areas.

Prior to the pandemic, an uneven number of doctors being rostered to run clinics across the sessions in a week resulted in significant peaks and troughs in patient attendance numbers. For example, in our large general ophthalmology outpatient clinic, the number of appointment slots for each half-day session varied between 107 and 195 . This led to periods of crowding in certain clinics waiting for areas at certain times. To meet safe distancing requirements and maximize patient throughput, the "load levelling" of scheduled patient attendances was necessary. Also, our Health Insights data analytics unit gathered information on patient loads and attendance at various times and generated "heat maps" to guide policy and decision-making. By analysing these maps, peak times when crowds were 
Fig. 5 Additional waiting areas created at a closed-off entrance and along the corridor. "Safety Ambassadors" patrol the various waiting areas to watch for over-crowding and advise patients to maintain safe distancing.

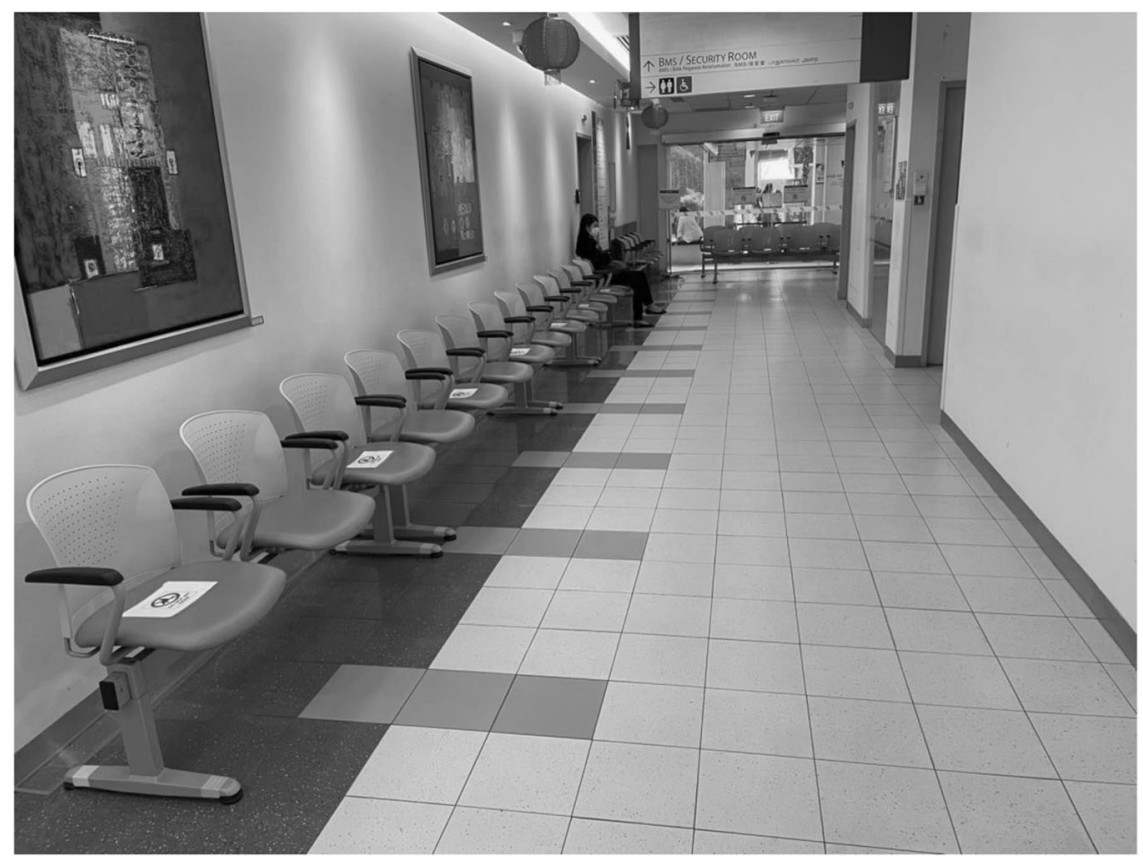

Fig. 6 Facilitating movement of patients. Signage to direct patients to new waiting areas and use of mobile screens for displaying patient queue numbers at these areas. observed at various touch points of the patient journey (such as at registration counters, investigation rooms, clinics and pharmacy) were identified (Fig. 7). Adjusting gaps between patient appointment times during these windows reduced crowding.

While load levelling is conceptually straightforward, its implementation was complex and tedious. This was because the uneven doctor workforce distribution was the result of years of accumulated changes to clinic rosters. To achieve effective load levelling in all of SNEC's clinics and satellites, the staffing roster of every doctor had to be redrawn. Following this, the patient appointment templates of every clinic were updated in the computerized appointment system, and then patient appointments moved to achieve levelling of the number of patients per session. Given a large number of doctors in SNEC and the thousands of patients with pre-existing appointments, this became a mammoth task, which required the full-time commitment of our administrative clinical service and resource management teams. 
Fig. 7 Using data analytics to minimize crowding. Example of a heat map indicating windows within which crowding was observed, and those with the capacity to accommodate more patients.

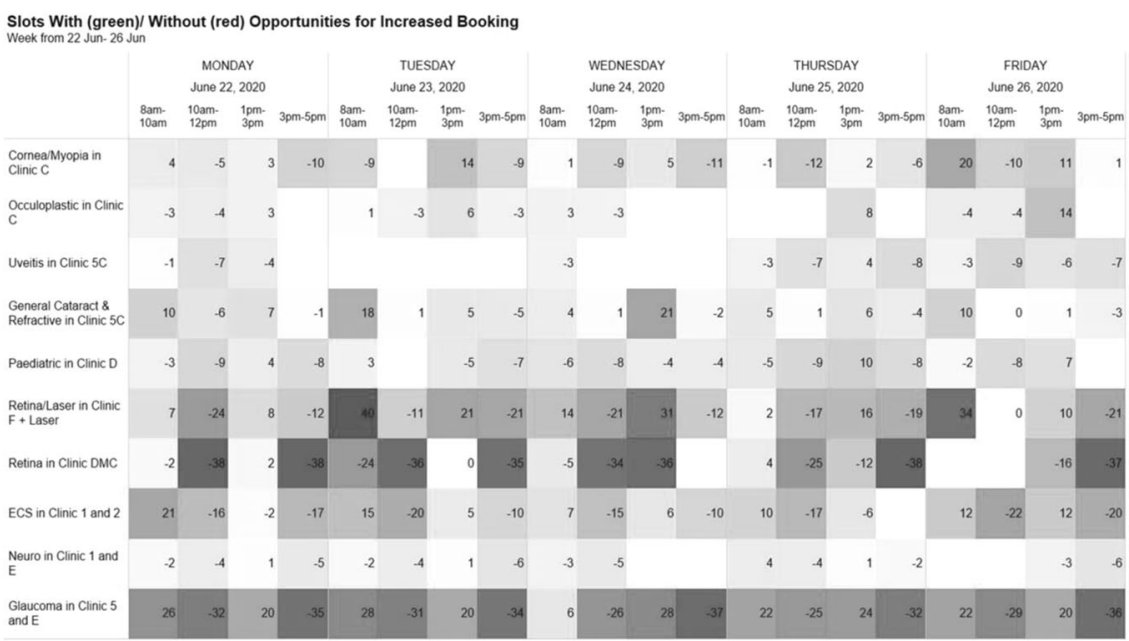

\section{Maximizing time}

In addition to optimizing the use of clinic space, another avenue of returning patient throughput to pre-pandemic levels while maintaining safe distancing was to extend the operating hours of the centre, which previously had clinic hours of 8.30 a.m. to 12.30 p.m. and 1.30 p.m. to 5.30 p.m., from Monday to Friday. Increasing clinic hours enabled an increase in the total number of patients seen each week, without overcrowding. Several subspecialties began running weekend clinics to cater for this need, supported by staff who were rostered to work on certain Saturdays. These new arrangements posed unique problems, as for many staff, working outside of normal hours was not part of their original employment terms, and consultation with the human resource department as well as individual negotiations with staff was required to permit alterations to previous working hours arrangements. In certain areas, administrative staff was rostered to help out in work that did not require nursing or medical skills, such as in entry point screening and crowd control in the patient waiting spaces. Manpower was also augmented through the hiring of temporary staff, some of whom previous employment was affected by the pandemic, to support clinic staff in their duties.

Maintaining a high level of senior management engagement has been a key strategy in managing our staff's well-being during these times of uncertainty and change. Through frequent communications, in the form of virtual town hall updates, email broadcasts as well as during key meetings, emphasis has been put on reinforcing the center's key missions, recognizing staff's dedication and contributions, keeping staff abreast of the latest developments, as well as reaching out to those who may need support.

\section{Minimizing physical attendances}

Much has been written on the use of technology to perform virtual or remote consultations, negating the need for patients to travel to the clinic [17-21]. Accelerated transition to these new models of care that are less dependent on physical patient attendance has become a key strategy for SNEC. Before the onset of the pandemic, the retinal and glaucoma services (the two largest departments) had already begun implementing virtual observation clinics for certain groups of patients, such as those with age-related macular degeneration or glaucoma suspects. In the phased reopening of services, these virtual observation clinics have been rapidly expanded in terms of capacity and indications. Other ophthalmic sub-specialties like neuro-ophthalmology and paediatric ophthalmology have also adopted similar models. Efforts are also underway to develop off-site facilities for ophthalmological investigations that patients can attend prior to their virtual or remote consultations, further reducing patient dwell time within SNEC.

\section{Outcomes and plans for the new normal}

By instituting the above measures, SNEC achieved a return to pre-pandemic levels of clinical load in 3 months and went on to exceed it in the following months, while meeting stringent government restrictions on safe distancing. Clinical load achieved in the months of September-November 2020 represented $113 \%$ of the corresponding period in 2019 (Fig. 1). Part of this is attributable to the expansion of the virtual clinics that now averages 281 cases per month compared to 100 per month during the pre-pandemic period (Fig. 8). At the same time, we were also able to achieve and surpass pre-pandemic surgical numbers (Fig. 3) through 
Fig. 8 Number of virtual consultations conducted. Dark bars: consultations performed. Light bars: consultations scheduled.

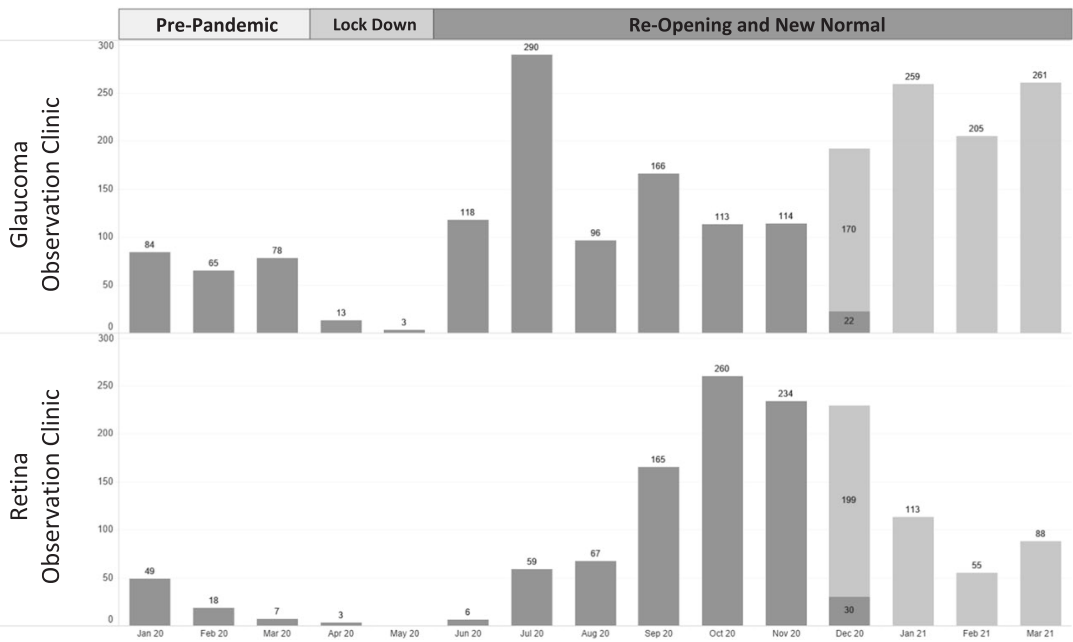

maximizing space use in our day-surgery ward and reducing patient dwell times.

While we have achieved a timely return to normal clinical throughput, there remains a risk of return to a lockdown state, as we have seen in countries and major cities affected by recurring waves of infections after initial containment measures were lifted. It is therefore important to plan ahead and establish contingency plans that can be quickly executed. From an operational perspective, planning can be guided by the interplay between three factors that would vary with each phase of pandemic response: (1) the stringency of mandated safe distancing measures; (2) the "availability" of patients; and (3) the availability of the medical workforce. During a lockdown, population movement is highly restricted. Patients with pre-existing appointments require timely deferral, and plans to execute a mass deferment must be developed beforehand. Precategorization of patients and clearly defining the roles and processes of different agencies during contingency plan activation will expedite the accomplishment of this task. Simultaneously, the need to roster more resources for urgent or "walk-in" patients should be anticipated. Strict distancing measures can also be expected, and plans prepared.

In a situation where the medical workforce is committed to supporting pandemic response operations external to their institution, workforce availability may become the limiting factor to the resumption of services. An appropriate strategy would be to attempt to maximize patient throughput as far as a medical resource and distancing measures allow, so as to minimize adding to the backlog of deferred patient appointments that accumulates during a lockdown. When the workforce has returned, institutions should continue to maximize patient throughput with the aim of clearing any backlog of cases and building spare capacity. In doing so, institutions will better position themselves to manage subsequent waves of outbreaks.
Common to all phases of this timeline should be the continual effort to implement new models of care to improve healthcare delivery and enhance value. This pandemic has provided an opportunity to accelerate these efforts as the impetus to develop them has become clearer and stronger. There is also greater understanding and acceptance by patients in receiving care through nontraditional means during this period. SNEC is currently in the process of setting up our Community Investigation Unit, a high-throughput off-site facility for the conduct of ophthalmological investigations, which is designed to significantly increase our virtual clinics' capacity.

\section{Discussion}

In this paper, we focused on the operational transformation of a large tertiary ophthalmic referral centre to tackle the difficulties posed by the pandemic. While our measures were largely in line with reports from other ophthalmic centres around the world, there was a difference in how we dealt with the anticipated increase in the need for emergency ophthalmic services. Our approach to this expected problem was to divert senior specialist manpower and resources towards these emergency cases. Some European centres took a different approach, leveraging on direct teleconsultation platforms to deal with the increase in emergency case load [19, 21]. Wickham et al. reported that using a teleconsult-first approach to emergency cases allowed them to manage $57 \%$ of cases without an initial physical consult [21]. Similarly, Bourdon et al. employed a teleconsult-first approach and required physical consults for only $27 \%$ of their first 500 patients [19]. These are examples of how accelerating the development of new models of care can help to tackle some of the issues brought on by the pandemic. In our centre, we also expanded our existing 
teleconsult and virtual consult platforms, but in contrast, focused on deploying these platforms for the provision of care to our existing stable glaucoma and retina service patients, replacing the need for physical visits in these clinics.

Although our responses allowed us to effectively and drastically reduce physical patient visits during the lockdown period, it is not clear how these reductions in physical visits impacted patient outcomes and safety. Clearly, the need to comply with public health restrictions and minimize the risk of COVID-19 transmission needs to be balanced against the visual morbidity and treatment outcomes of our patients. Longer-term patient outcomes and safety data as a result of operational responses to the pandemic are an area deserving of future study. Related to this, Wickham et al. report lower presentation rates for various sight-threatening conditions, such as retinal detachments, during the pandemic lockdown period, and these delayed presentations are likely to have long-lasting effects on patient visual outcomes and disease burden, that is also an area deserving of further study [21].

Lastly, although we achieved a rapid return to prepandemic operational throughput, the backlog of patient appointments that was created from deferred visits during the lockdown would take months to clear. This has manifested as extended appointment waiting times for nonurgent new patient referrals to our centre. Initiatives like the expansion of virtual clinics and our Community Investigation Unit would serve to reduce the need for patient deferments should subsequent lockdowns be instituted. However, this effect would be relatively small. As such, while we are now better prepared to safely and rapidly reduce patient visits if again required, the return to normalcy would take longer after each outbreak resurgence.

\section{Conclusion}

Here we have outlined SNEC's operational response to Singapore's COVID-19 lockdown measures, phased reopening and movement towards the new normal. It is prudent to think ahead and develop plans to respond to possible resurgences of COVID-19. In the midst of this unprecedented event, opportunities to review and revamp past systems have arisen, and the impetus for developing new models of care has become much stronger. Operational and administrative staff have played major roles in SNEC's response to the crisis. While their work may not be as visible as that of medical personnel deployed to the frontlines [22], it is equally important. Their ability to quickly plan and execute various complex and demanding tasks continues to be key to ensuring the safety of the center's staff and maintaining an appropriate level of care for our patients. In the months and years ahead, there will be a need to continue to develop and strengthen these capabilities as we head towards an increasingly uncertain future.

\section{Summary}

\section{What was known before}

- Infection control and clinical measures for ophthalmic institutions and departments to cope with the COVID-19 pandemic.

\section{What this study adds}

- Detailed implementation plans to manage lockdowns and subsequent re-openings. Specific operational responses and their outcomes.

Author contributions HBL was responsible for the overall design and writing of the manuscript. T-ET and BJF contributed in the interpretation of results and drafting of the paper. $\mathrm{JH}$ was responsible for performing data analytics and producing the tables and charts. AA, WBK and PYB were responsible for extraction and analyses on data from the satellite clinics. SYL and EYMW provided feedback on the manuscript. TYW provided feedback and overall guidance on the writing of the article.

\section{Compliance with ethical standards}

Conflict of interest The authors declare no competing interests. No funding has been received by any of the authors for the writing of this article.

Publisher's note Springer Nature remains neutral with regard to jurisdictional claims in published maps and institutional affiliations.

\section{References}

1. World Health Organization. WHO characterizes COVID-19 as a pandemic. World Health Organization. 2020. https://www.who. int/emergencies/diseases/novel-coronavirus-2019/events-as-theyhappen. Accessed 19 Apr 2020.

2. World Health Organization. Coronavirus Disease (COVID-19) situation reports. https://www.who.int/emergencies/diseases/ novel-coronavirus-2019/situation-reports. Accessed 1 May 2020.

3. Pung R, Chiew CJ, Young BE, Chin S, Chen MI-C, Clapham HE, et al. Investigation of three clusters of COVID-19 in Singapore: implications for surveillance and response measures. Lancet. 2020;395:1039-46.

4. Singapore Government Portal. Strong national push to stem spread of COVID-19. https://www.moh.gov.sg/news-highlights/ details/strong-national-push-to-stem-spread-of-covid-19. Accessed 26 Apr 2020. 
5. Khor WB, Yip L, Zhao P, Foo VHX, Lim L, Ting DSW, et al. Evolving practice patterns in Singapore's Public Sector Ophthalmology Centers During the COVID-19 Pandemic. Asia Pac J Ophthalmol. 2020;9:285-90.

6. Borrelli E, Sacconi R, Querques L, Zucchiatti I, Prascina F, Bandello F, et al. Taking the right measures to control COVID-19 in ophthalmology: the experience of a tertiary eye care referral center in Italy. Eye. 2020;34:1175-6.

7. Cheung SSL, Wong CYK, Chan JCK, Chan CKM, Lam NM, Yuen HKL, et al. Ophthalmology in the time of COVID-19: experience from Hong Kong Eye Hospital. Int $\mathbf{J}$ Ophthalmol. 2020;13:851-9.

8. Jeong A, Sagong M. Changes in the clinical practice of ophthalmology during the Coronavirus Disease 2019 (COVID-19) Outbreak: an experience from Daegu, Korea. Infect Chemother. 2020;52:226-30.

9. Lai THT, Tang EWH, Chau SKY, Fung KSC, Li KKW. Stepping up infection control measures in ophthalmology during the novel coronavirus outbreak: an experience from Hong Kong. Graefes Arch Clin Exp Ophthalmol. 2020;258:1049-55.

10. Lim LW, Yip LW, Tay HW, Ang XL, Lee LK, Chin CF, et al. Sustainable practice of ophthalmology during COVID-19: challenges and solutions. Graefes Arch Clin Exp Ophthalmol. 2020;258:1427-36.

11. Ma X, Lin J, Fang S. Precautions in ophthalmic practice in a hospital with the risk of COVID-19: experience from China. Acta Ophthalmol. 2020;98:e520-1.

12. Romano MR, Montericcio A, Montalbano C, Raimondi R, Allegrini D, Ricciardelli G, et al. Facing COVID-19 in ophthalmology department. Curr Eye Res. 2020;45:653-8.

13. Safadi K, Kruger JM, Chowers I, Solomon A, Amer R, Aweidah $\mathrm{H}$, et al. Ophthalmology practice during the COVID-19 pandemic. BMJ Open Ophthalmol. 2020;5:e000487.
14. Shih CK, Chan JCH, Lai JSM. Maintenance of ophthalmic specialist out-patient service during the COVID-19 outbreak: The University of Hong Kong experience. Eye. 2020;34:1241-2.

15. Yu A-Y, Tu R, Shao X, Pan A, Zhou K, Huang J. A comprehensive Chinese experience against SARS-CoV-2 in ophthalmology. Eye Vis. 2020;7:19.

16. Ministry of Health, Singapore. Continuation of Essential Healthcare Services during Period of Heightened Safe Distancing Measures. Ministry of Health. 2020. https://www.moh.gov.sg/newshighlights/details/continuation-of-essential-healthcare-servicesduring-period-of-heightened-safe-distancing-measures. Accessed 3 May 2020.

17. Ting DS, Gunasekeran DV, Wickham L, Wong TY. Next generation telemedicine platforms to screen and triage. $\mathrm{Br} \mathrm{J}$ Ophthalmol. 2020;104:299-300.

18. Ting DS, Carin L, Dzau V, Wong TY. Digital technology and COVID-19. Nat. Med. 2020;26:459-61.

19. Bourdon H, Jaillant R, Ballino A, El Kaim P, Debillon L, Bodin S, et al. Teleconsultation in primary ophthalmic emergencies during the COVID-19 lockdown in Paris: Experience with 500 patients in March and April 2020. J Fr Ophtalmol. 2020;43:577-85.

20. Liebmann JM. Ophthalmology and glaucoma practice in the COVID-19 era. J Glaucoma. 2020;29:407-8.

21. Wickham L, Hay G, Hamilton R, Wooding J, Tossounis H, da Cruz L, et al. The impact of COVID policies on acute ophthalmology services-experiences from Moorfields Eye Hospital NHS Foundation Trust. Eye. 2020;34:1189-92.

22. Coronavirus: The healthcare workers who answer the call for front-line volunteers. Straits Times. 2020. https://www.stra itstimes.com/singapore/coronavirus-many-healthcare-workers-a nswering-the-call-for-frontline-volunteers. Accessed Apr 2020. 